\title{
Inhomogeneous distribution of mercury on the surfaces of rapidly rotating HgMn stars
}

\author{
O. Kochukhov ${ }^{1}$, N. Piskunov ${ }^{1}$, M. Sachkov ${ }^{2}$, and D. Kudryavtsev ${ }^{3}$ \\ 1 Department of Astronomy and Space Physics, Uppsala University, 75120 Uppsala, Sweden \\ e-mail: oleg@astro.uu.se \\ 2 Institute of Astronomy, Russian Academy of Sciences, Pyatnitskaya 48, 119017 Moscow, Russia \\ 3 Special Astrophysical Observatory, Russian Academy of Sciences, Nizhnij Arkhyz, 357147 Karachai-Cherkessian \\ Republic, Russia
}

Received 24 March 2005 / Accepted 26 April 2005

\begin{abstract}
Starspots are usually associated with the action of magnetic fields at stellar surfaces. However, an inhomogeneous chemical distribution of mercury was found recently for the mercury-manganese ( $\mathrm{HgMn})$ star $\alpha$ And - a well-established member of a non-magnetic subclass of the chemically peculiar stars of the upper main sequence. In this study we present first results of the high-resolution survey of the Hg II $3984 \AA$ A resonance line in the spectra of rapidly rotating HgMn stars with atmospheric parameters similar to those of $\alpha$ And. We use spectrum synthesis modelling and take advantage of the Doppler resolution of stellar surfaces to probe the horizontal structure of mercury distribution. Clear signatures of spots are found in the Hg II $3984 \AA$ line profiles of HR 1185 and HR 8723. Two observations of the latter star separated by two days give evidence for line profile variability. We conclude that inhomogeneous distribution of $\mathrm{Hg}$ is a common phenomenon for the rapidly rotating $\mathrm{HgMn}$ stars in the 13000-13800 K effective temperature range independent of the stellar evolutionary stage. These results establish the existence of a new class of spectrum variable spotted B-type stars. It is suggested that the observed $\mathrm{Hg}$ inhomogeneities arise from dynamical instabilities in the chemical diffusion processes and are unrelated to magnetic phenomena.
\end{abstract}

Key words. line: profiles - stars: atmospheres - stars: chemically peculiar - stars: individual: HR 1185 stars: individual: HR 8723

\section{Introduction}

In several classes of the main sequence stars, surface structures are created and supported by a strong magnetic field. The Sun and late-type active stars show complex surface magnetic topologies where the field suppresses convective energy transport and creates cool dark spots. These structures evolve on a relatively short timescale, whereas the magnetic field itself is regenerated by a contemporary dynamo mechanism and undergoes quasi-periodic evolution during activity cycles lasting from a few years to decades. In contrast, some of the B, $\mathrm{A}$, and $\mathrm{F}$ chemically peculiar stars have strong global, often dipolar, fields of $\sim 1-10 \mathrm{kG}$ strength, which do not change on short timescales and are believed to be fossil remnants from the epoch of stellar formation. These strong fields introduce anisotropy in the processes of radiative diffusion, resulting in depletion and accumulation of certain chemical elements in different depth layers and surface zones (Michaud et al. 1981). Since magnetic field geometry and uneven surface chemical distribution are generally non-symmetric with respect to the rotation axis, many upper main sequence magnetic stars exhibit rotational modulation of various magnetic field observables, line profiles, and brightness in broad photometric bands - a behaviour commonly described by the oblique rotator model.

Alongside the magnetic spotted chemically peculiar stars, there exists a group of mercury-manganese ( $\mathrm{HgMn})$ stars for which no solid evidence of a magnetic field is detected (Shorlin et al. 2002, and references therein). These objects are found within the main sequence band in the $T_{\text {eff }}$ range between 10500 and $15000 \mathrm{~K}$ and have extremely stable photospheres, providing one of the best astrophysical laboratories for investigation into radiative diffusion in stars. The variability phenomena associated with the oblique rotator framework have been neither unambiguously detected nor expected for the $\mathrm{HgMn}$ stars until recently. This paradigm had to be abandoned when convincing evidence for profile changes in the resonance $\mathrm{Hg}$ II $3984 \AA$ A line was revealed by the very high quality observations of the brightest $\mathrm{HgMn}$ star $\alpha$ And collected by Ryabchikova et al. (1999) and Adelman et al. (2002). The authors of the latter study demonstrate that the most likely interpretation of this variability is an inhomogeneous $\mathrm{Hg}$ surface distribution, coupled with the 2.382 day rotation period of the star. Doppler mapping of the mercury distribution in $\alpha$ And (Adelman et al. 2002) showed that this element is depleted 
close to both rotation poles and is concentrated in a series of spots located at the stellar equator.

The discovery of $\mathrm{Hg}$ spots in the atmosphere of $\alpha$ And has challenged our understanding of the nature of $\mathrm{HgMn}$ stars and of the role of magnetic fields in creating stellar surface structures. In no classical magnetic A or B star with inhomogeneous chemical distributions could we find a spotted structure limited to just one chemical element and showing such a high degree of symmetry with respect to the stellar rotation axis. Adding to this puzzle, numerous high-precision spectropolarimetric observations of $\alpha$ And (Glagolevskij et al. 1985; Chountonov 2001; Wade et al. 2004) have failed to detect a magnetic field which could be responsible for the observed anomalous behaviour of $\mathrm{Hg}$.

A clue to the nature of the $\alpha$ And phenomenon can be obtained by establishing its extent among other HgMn stars. This requires collecting high-resolution high $S / N$ observations of $\mathrm{Hg}$ lines followed by a careful time-series analyses of the integral line profile characteristics, such as equivalent width, line depth, and central wavelength. A different and more sensitive type of observation can be performed for the rapidly rotating HgMn stars. In these objects the stellar line shapes are dominated by the rotational Doppler broadening, and spectral contribution of different surface zones could be resolved in the profiles of absorption lines. In this situation surface structures manifest themselves through significant deviation of the spectral line shapes from a pure rotational profile. The Doppler imaging method (Vogt \& Penrod 1983) exploits these unique properties of the spectra of rapidly rotating stars and should be capable of establishing the presence of stellar surface inhomogeneities from a single high-quality recording of a spectral line profile.

In the present study we utilize the Doppler imaging principle and search for the signatures of mercury spots in the atmospheres of rapidly rotating $\mathrm{HgMn}$ stars with $T_{\text {eff }}$ similar to that of $\alpha$ And. Using this approach we discover two HgMn stars with inhomogeneous surface distribution of $\mathrm{Hg}$ and demonstrate line profile variability for one of them.

\section{Observations and data reduction}

We recorded high-resolution spectra of rapidly rotating HgMn stars using the cross-dispersed Nasmyth Echelle Spectrometer (NES, Panchuk et al. 1999) installed at the 6-m telescope of the Russian Special Astrophysical Observatory. A sample of 6 bright $(V=5.45-6.05) \mathrm{HgMn}$ stars was chosen according to the spectral classification in the SIMBAD database and from literature references. Catalogues of the projected rotational velocity measurements (Abt et al. 2002; Strom et al. 2003) were used to select $\mathrm{HgMn}$ stars with $v_{\mathrm{e}} \sin i \gtrsim 50 \mathrm{~km} \mathrm{~s}^{-1}$. According to the literature references in SIMBAD and information in the relevant catalogues (Pourbaix et al. 2004), none of the program stars is known to be a spectroscopic binary.

Stellar observations were obtained during the period between July 28 and August 5, 2004. All program stars were observed once, except HR 8723 for which two spectra were obtained. A $2 \mathrm{~K} \times 2 \mathrm{~K}$ Loral $\mathrm{CCD}$ detector used with the
Table 1. The log of NES observations of rapidly rotating HgMn stars. Columns give the HR and HD numbers of the target stars, heliocentric Julian Date for the middle of exposure, the length of exposure, and the signal-to-noise ratio achieved for the spectral region around the $\mathrm{Hg}$ II $3984 \AA$ A line.

\begin{tabular}{ccccc}
\hline \hline HR & HD & HJD-2 400 000 & $T_{\text {exp }}(\min )$ & $S / N$ \\
\hline 1079 & 21933 & 53223.506 & 50 & 290 \\
1185 & 23950 & 53218.519 & 55 & 370 \\
1445 & 28929 & 53215.532 & 60 & 330 \\
1484 & 29589 & 53219.548 & 40 & 330 \\
1576 & 31373 & 53224.523 & 60 & 290 \\
8723 & 216831 & 53216.520 & 40 & 510 \\
& & 53218.432 & 40 & 470 \\
\hline
\end{tabular}

NES spectrograph allowed us to record 22 echelle orders with complete wavelength coverage of the 3495-4190 A region and with a spectral resolving power of $\lambda / \Delta \lambda=36500$.

The software package REDUCE (Piskunov \& Valenti 2002) was used for the optimal extraction of the NES spectra. A wavelength scale with an internal accuracy of $50-70 \mathrm{~m} \mathrm{~s}^{-1}$ was established by means of a 2-D wavelength calibration procedure which made use of $\approx 600$ ThAr lines in all echelle orders. Provisional normalization of the spectra was performed by fitting a smooth function to the line-free spectral regions. This procedure is not applicable to the echelle orders containing wide hydrogen Balmer lines. Continuum in these regions was instead adjusted in such a way that the observed hydrogen line wings would match synthetic spectra computed as described in Sect. 3.

For the employed CCD detector and spectral resolution of our observations the instrumental profile of the spectrograph is somewhat oversampled (4.4 pixel/resolution element). This allowed us to reduce the pixel sampling of the spectra by a factor of 2, which increased the $S / N$ ratio without compromising resolution. The signal-to-noise ratio of the resampled spectra lies in the range of $290-510$ for the spectral region containing the Hg II $3984 \AA$ line.

The summary of our spectroscopic observations of $\mathrm{HgMn}$ stars is given in Table 1.

\section{Spectrum synthesis calculations}

To probe the structure of mercury distribution on the surfaces of HgMn stars we had to develop an objective method for characterizing deviation of the observed profile of the resonance Hg II line at $\lambda 3984 \AA$ from the basic rotational profile. We chose to compare the observed spectra with the theoretical spectrum synthesis computation performed under assumption of a homogeneous $\mathrm{Hg}$ abundance distribution.

The colours in the Strömgren photometric system were extracted from the $u v b y \beta$ catalogue of Hauck \& Mermilliod (1998) and were used to determine atmospheric parameters, $T_{\text {eff }}$ and $\log g$, with the help of two widely used photometric calibrations (Moon \& Dworetsky 1985; Napiwotzki et al. 1993) implemented in the TEMPLOGG code (Rogers 1995). For all 
Table 2. Atmospheric parameters and other characteristics of the rapidly rotating HgMn stars included in our study. Columns give the HR number of the program stars, effective temperature and surface gravity obtained from the Strömgren photometric colours using calibrations of Moon $\&$ Dworetsky (1985) and Napiwotzki et al. (1993), $T_{\text {eff }}$ and $\log g$ adopted in the present paper, Hipparcos parallax, and projected rotational velocity. The last three columns report inferred abundances of $\mathrm{Si}, \mathrm{Y}$, and $\mathrm{Hg}$. The latter parameter corresponds to the optimum fit of the $\mathrm{Hg}$ II line wings.

\begin{tabular}{|c|c|c|c|c|c|c|c|c|c|c|c|}
\hline \multirow[t]{2}{*}{ HR } & \multicolumn{2}{|c|}{ Moon \& Dworetsky (1985) } & \multicolumn{2}{|c|}{ Napiwotzki et al. (1993) } & \multicolumn{2}{|c|}{ Adopted } & \multirow{2}{*}{$\begin{array}{c}v_{\mathrm{e}} \sin i \\
\left(\mathrm{~km} \mathrm{~s}^{-1}\right)\end{array}$} & \multirow{2}{*}{$\begin{array}{c}\pi \\
\text { (mas) }\end{array}$} & \multicolumn{3}{|c|}{$\log N / N_{\text {tot }}$} \\
\hline & $T_{\text {eff }}(\mathrm{K})$ & $\log g$ & $T_{\text {eff }}(\mathrm{K})$ & $\log g$ & $T_{\text {eff }}(\mathrm{K})$ & $\log g$ & & & $\mathrm{Si}$ & $\mathrm{Y}$ & $\mathrm{Hg}$ \\
\hline 1079 & 11948 & 4.13 & 12040 & 4.07 & 12000 & 4.1 & 87.6 & $9.18 \pm 0.87$ & -5.00 & -6.60 & -6.00 \\
\hline 1185 & 12892 & 4.06 & 13110 & 3.97 & 13000 & 4.0 & 67.0 & $10.14 \pm 0.90$ & -4.80 & -7.50 & -5.45 \\
\hline 1445 & 12885 & 4.05 & 12990 & 3.97 & 12900 & 4.0 & 56.4 & $7.00 \pm 0.86$ & -4.40 & -7.80 & -6.15 \\
\hline 1484 & 14473 & 4.29 & 14763 & 4.16 & 14600 & 4.2 & 63.2 & $9.46 \pm 0.78$ & -4.70 & -7.50 & -6.80 \\
\hline 1576 & 13884 & 4.14 & 14013 & 4.03 & 13900 & 4.1 & 74.6 & $7.71 \pm 0.87$ & -4.50 & -7.50 & -6.20 \\
\hline 8723 & 13057 & 3.65 & 13058 & 3.56 & 13100 & 3.6 & 67.1 & $3.90 \pm 0.70$ & -4.65 & -7.30 & -6.40 \\
\hline
\end{tabular}

program stars we found consistent atmospheric parameters and adopted effective temperature and surface gravity in between the values given by the two calibrations (see Table 2). The errors in $T_{\text {eff }}$ and $\log g$ are estimated to be $200 \mathrm{~K}$ and $0.1 \mathrm{dex}$, respectively. Model atmospheres of the program stars were generated with the ATLAS9 code of Kurucz (1993) assuming zero microturbulence and solar chemical composition.

The spectrum synthesis calculations in this paper were carried out with the help of the SYNTH3 code developed by O. Kochukhov. This program is based on the SYNTH code (Piskunov 1992) and SME package (Valenti \& Piskunov 1996). SYNTH3 uses a non-magnetic version of the quadratic DELO radiative transfer algorithm (Socas-Navarro et al. 2000) to compute theoretical intensity spectra on the adaptive wavelength grid for a range of angles between the line of sight and normal to the stellar surface. The disk-integrated flux spectrum is calculated for a given $v_{\mathrm{e}} \sin i$ by summing the Doppler-broadened contributions of different surface annular zones. This spectrum synthesis approach allows us to reach higher precision in modelling the spectra of rapidly rotating stars compared to the convolution of the flux spectra with a Doppler broadening function (Gray 1992) that assumes a linear limb-darkening law.

Numerical procedure for the calculation of the opacity due to the hydrogen Balmer lines implemented in SYNTH3 includes recent developments in the Balmer line broadening theory (Stehlé 1994; Barklem et al. 2000).

The atomic line list used in our study of the HgMn stars was obtained from the VALD database (Kupka et al. 1999). In addition, the line list was complemented by 15 isotope and hyperfine components of the $\mathrm{Hg}$ II $3984 \AA$ line based on the data given in Table 3 of Woolf \& Lambert (1999). The oscillator strengths of the $\mathrm{Hg}$ II line components were recalculated for the terrestrial isotope mixture of $\mathrm{Hg}$ (see Table 1 in Proffitt et al. 1999).

\subsection{Si Il lines}

Since all $\mathrm{HgMn}$ stars investigated here are characterized by substantial rotation, $v_{\mathrm{e}} \sin i$ is the most important parameter determining the spectral line shapes. Accurate measurements of the projected rotational velocity were obtained by fitting a short spectral segment containing strong Si II lines at $\lambda$ 4128.05, $4130.87,4130.89 \AA$. The stellar radial velocity, $v_{\mathrm{e}} \sin i$, and the silicon abundance were adjusted simultaneously. Occasionally, concentrations of $\mathrm{Mn}, \mathrm{Ti}$, and $\mathrm{Cr}$ were also modified to obtain a better description of the weak features of these elements blending the $\mathrm{Si}$ II lines. Resulting $v_{\mathrm{e}} \sin i$ parameter and $\mathrm{Si}$ abundance are reported in Table 2 . These values are uncertain to approximately $\pm 1 \mathrm{~km} \mathrm{~s}^{-1}$ and \pm 0.05 dex, respectively.

The fit of the high $S / N$ spectra of HgMn stars in the 4126-4134 $\AA$ region is illustrated in Fig. 1. For all 7 observations of 6 stars we are able to obtain a very good description of the Si II line shapes. No distortion of the Si II lines indicative of the surface structures or spectroscopic binarity is detected.

\subsection{Hg II line}

The resonance line of $\mathrm{Hg}$ II at $\lambda 3984 \AA$ was analysed using the $v_{\mathrm{e}} \sin i$ value determined from fitting the Si II lines. To achieve a good description of this spectral region, abundances of $\mathrm{Hg}, \mathrm{Y}$, and $\mathrm{Mn}$ were adjusted simultaneously. Absorption due to the red wing of the hydrogen $\mathrm{H} \varepsilon$ line was also taken into account. Resulting best-fit synthetic spectra are illustrated in Fig. 2, while inferred abundances of $\mathrm{Hg}$ and $\mathrm{Y}$ are given in Table 2. The respective internal error bars are approximately 0.1 and 0.3 dex. For HR 1445, HR 1484, HR 1079, and HR 1576 the observed spectra can be reproduced within the error bars with a unique $\mathrm{Hg}$ abundance. We note that the double structure visible in the $\mathrm{Hg}$ II line of HR 1079 is due to the blending with the unusually strong Y II $3982.59 \AA$ line and is successfully modelled by our theoretical calculation. At the same time, we find that the $\mathrm{Hg}$ II line in the spectra of HR 1185 and HR 8723 could not be fitted by assuming a homogeneous surface distribution of mercury. When $\mathrm{Hg}$ abundance is chosen to describe the outer line wings, the inner part of the profile is too deep compared to observations. Decreasing abundance to fit intensity in the line core does not produce acceptable results either. It is clear that the overall shape of the Hg II $3984 \AA$ line deviates significantly from the Doppler-broadened profile expected for these two HgMn stars. 


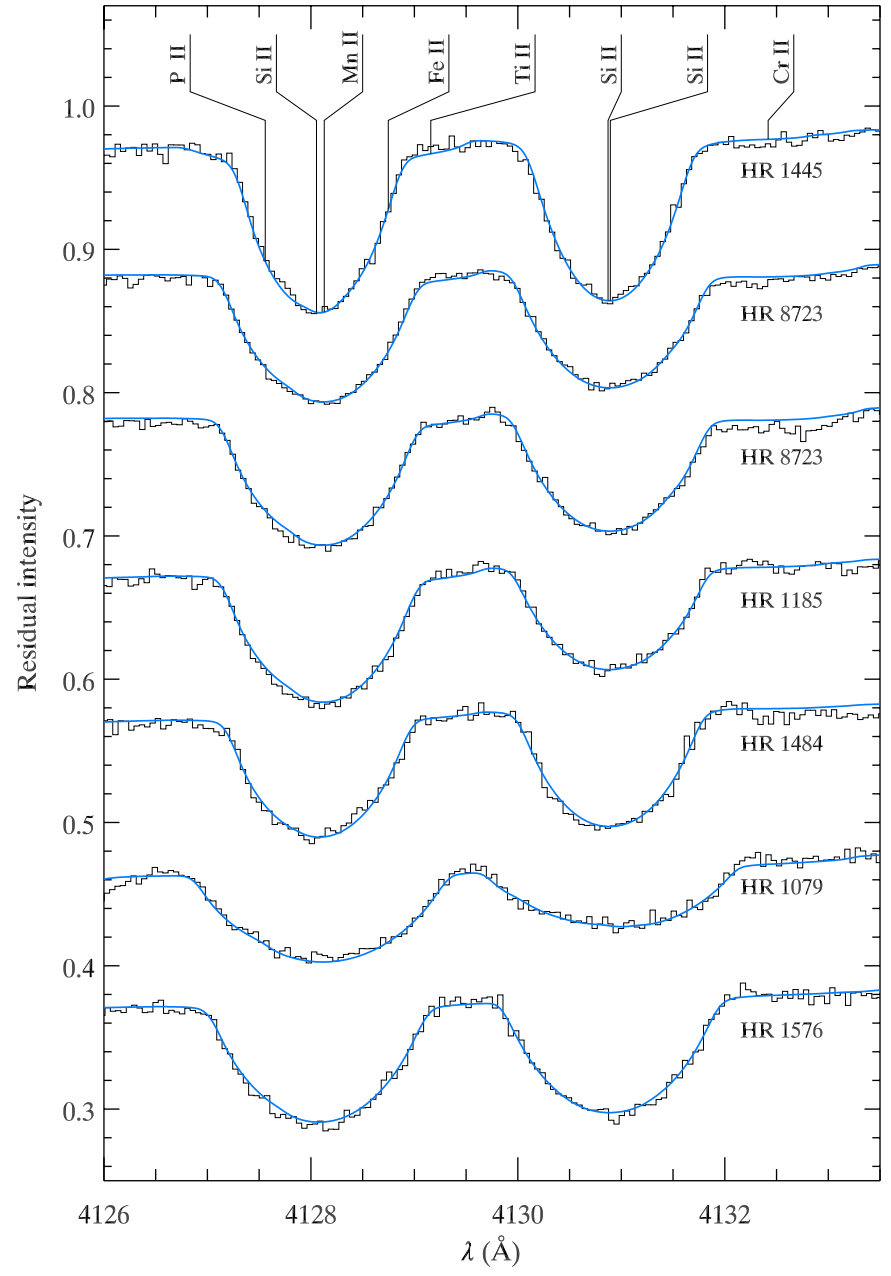

Fig. 1. The spectra of rapidly rotating HgMn stars in the vicinity of the Si II 4128.05 and $4130.89 \AA$ lines. Observations are shown with histograms, whereas the solid curves correspond to the best-fit synthetic spectra. Stellar spectra are displaced in the vertical direction for display purposes. Two observations of HR 8723 are compared with the same theoretical calculation.

It should be emphasized that the wavelength separation of the isotope components of the $\mathrm{Hg}$ II line is considerably smaller than Doppler broadening for $v_{\mathrm{e}} \sin i=50-70 \mathrm{~km} \mathrm{~s}^{-1}$; hence, an unusual isotope mixture could not be responsible for the observed line shapes. Our spectrum synthesis calculations demonstrate that, even in the extreme case when the $\mathrm{Hg}$ II line contains only the ${ }^{196} \mathrm{Hg}$ and ${ }^{204} \mathrm{Hg}$ isotope components, a flatbottomed spectral line shape is only seen for $v_{\mathrm{e}} \sin i$ not exceeding $\approx 15 \mathrm{~km} \mathrm{~s}^{-1}$.

The shallow Hg II line profiles of HR 1185 and HR 8723 bear strong resemblance to the appearance of this line in some of the rotation phases of $\alpha$ And (see Fig. 5 in Adelman et al. 2002). Consequently, the line profile anomalies found for HR 1185 and HR 8723 are interpreted as a signature of an inhomogeneous surface distribution of $\mathrm{Hg}$.

Two observations of HR 8723 separated by 2 days are shown in Fig. 2. The $\mathrm{Hg}$ II line profile has clearly changed, whereas the Si II lines (Fig. 1) and all other spectral features are identical within the noise level in the two spectra. This behaviour is strikingly similar to the spectrum variability pattern

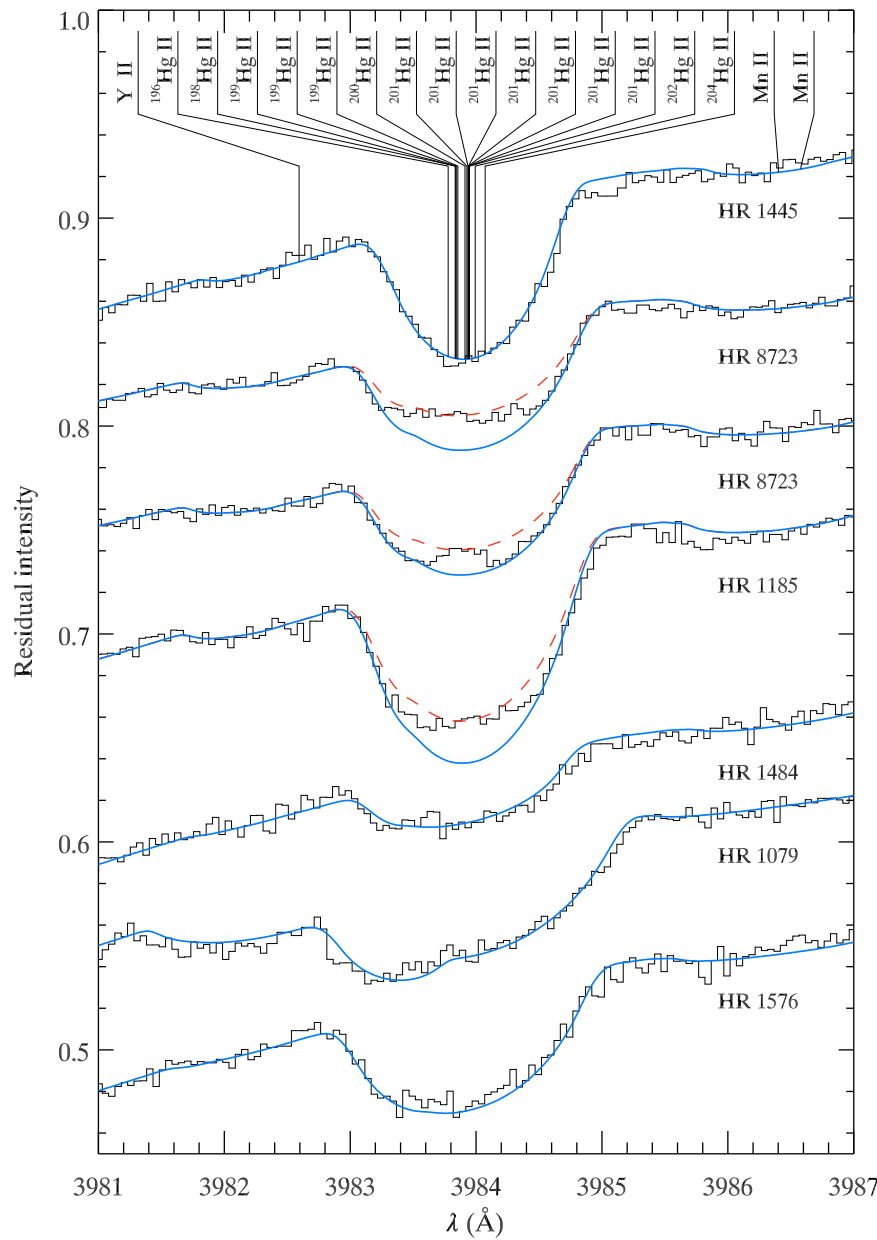

Fig. 2. The resonance $\mathrm{Hg}$ II $3984 \AA$ in the spectra of rapidly rotating HgMn stars. Observations are shown with histogram, whereas lines correspond to the best-fit theoretical spectra. The solid curve illustrates the fit of the $\mathrm{Hg}$ II line wings. For the spectrum of HR 1185 and two observations of HR 8723 showing anomalous line profile shapes, an additional theoretical spectrum (dashed line) shows an approximate fit of the line cores. The spectra of the HgMn stars are displaced in the vertical direction for display purposes.

of $\alpha$ And and strengthens conclusion that the $\mathrm{Hg}$ II line anomalies seen in HR 8723 arise from a spotted distribution of $\mathrm{Hg}$. Thus, we expect the corresponding profile variability to occur with the rotation period of the star.

\section{Discussion}

Inspired by the recent discovery and mapping of $\mathrm{Hg}$ spots in the atmosphere of $\alpha$ And (Adelman et al. 2002), we carried out a high $S / N$ survey of the Hg II $3984 \AA$ line in a carefully chosen group of rapidly rotating $\mathrm{HgMn}$ stars. For such objects, Doppler broadening of the stellar spectra allowed us to obtain partial resolution of the stellar surface and to probe possible $\mathrm{Hg}$ inhomogeneities. Our observations demonstrate that a spotted $\mathrm{Hg}$ distribution is not limited to $\alpha$ And and that it exists in other HgMn stars. With the help of detailed spectrum synthesis calculations, we revealed signatures of an inhomogeneous mercury distribution in two out of six studied stars. Furthermore, for the HgMn star HR 8723 we discover profile 


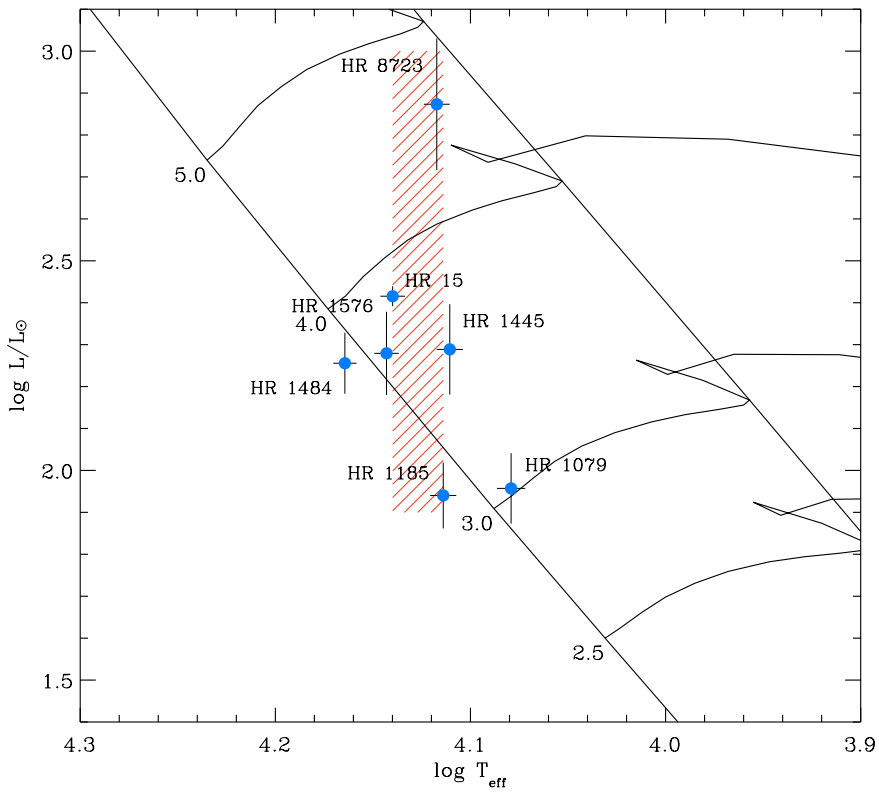

Fig. 3. Effective temperatures and luminosities of rapidly rotating HgMn stars (symbols) are compared with the theoretical evolutionary tracks of Schaller et al. (1992). The shaded area highlights the $13000-13800 \mathrm{~K} T_{\text {eff }}$ range where $\mathrm{HgMn}$ stars with an inhomogeneous mercury surface distribution have been found so far.

variability in the $\mathrm{Hg}$ II line. These observations establish the existence of a new class of spectrum variable, spotted, apparently non-magnetic, HgMn stars. The three currently known members include $\alpha$ And, HR 1185, and HR 8723.

Precise Hipparcos parallaxes (ESA 1997) reported for the program stars in Table 2 enable us to establish evolutionary status of the HgMn stars investigated here. In Fig. 3 we compare effective temperatures and luminosities of the $\mathrm{HgMn}$ stars studied by us and $\alpha$ And (=HR 15, $T_{\text {eff }}=13800 \mathrm{~K}$, Ryabchikova et al. 1999) with the theoretical stellar evolutionary tracks of Schaller et al. (1992). Most of the stars are located close to the zero-age main sequence line and have masses between 3.0 and $4.0 \mathcal{M}_{\odot}$. HR 8723 is apparently a more massive and more evolved $\mathrm{HgMn}$ star with $\mathcal{M} \approx 4.5 \mathcal{M}_{\odot}$. There seems to be no relation between the stellar age and presence of mercury spots. At the same time, one striking result is that the spotted $\mathrm{HgMn}$ stars occupy a relatively narrow range of $T_{\mathrm{eff}}$ between 13000 and $13800 \mathrm{~K}$. Further observations are required to confirm this intriguing behaviour; but taken at face value, the outcome of our study indicates that $\mathrm{Hg}$ spots can only form in the atmospheres of rapidly rotating $\mathrm{HgMn}$ stars lying in this limited $T_{\text {eff }}$ interval.

The origin of $\mathrm{Hg}$ inhomogeneities in the atmospheres of $\mathrm{HgMn}$ stars remains a mystery. The standard link between stellar surface structures and magnetic field is doubtful in this case because $\mathrm{HgMn}$ stars as a class are known to be non-magnetic (Shorlin et al. 2002). Numerous attempts to detect magnetic field in $\alpha$ And itself (Glagolevskij et al. 1985; Chountonov 2001; Wade et al. 2004) steadily decrease the upper limit on the strength of the possible global field, culminating in the recent unprecedented longitudinal field measurement consistent with zero magnetic field, with an error bar less than 10 Gauss, obtained with the new ESPaDOnS spectropolarimeter at CFHT by $\mathrm{G}$. Wade and collaborators (private communication). This places a stringent upper limit of a few tens of Gauss on the possible large-scale field, which is well below the equipartition limit for $\alpha$ And.

Furthermore, the high-resolution spectropolarimetric observations by Wade et al. (2004) also place severe constraints on the possible presence of higher-order multipole and tangled fields - a type of magnetic topology that has been successfully detected using identical observational methods in the Dopplerbroadened Stokes $V$ profiles of active stars (Donati et al. 1997). Thus, if we assume that the spotted $\mathrm{HgMn}$ stars are magnetic at some low level, their field topology, as well as the origin of magnetism, have to be very unusual and qualitatively different from both global fossil fields of the early-type chemically peculiar stars and complex dynamo-generated magnetic structures found in the active late-type stars. At the same time, these hypothetical magnetic fields of $\mathrm{HgMn}$ stars have to possess some degree of global coherency in order to be related to the observed large-scale $\mathrm{Hg}$ structures. At the current level of understanding it appears exceedingly difficult to reconcile these contradictory requirements on the field topology. Therefore, we consider a magnetic explanation of the $\mathrm{Hg}$ inhomogeneities to be very unlikely.

An alternative interpretation may be related to the possible dynamical instability of the diffusion processes in rapidly rotating $\mathrm{HgMn}$ stars. It is not inconceivable that in the narrow range of stellar parameters where $\mathrm{Hg}$ spots are found accumulation of this element in the line-forming region becomes extremely sensitive to external perturbations. In this situation a few $\%$ reduction of the effective gravitational acceleration at the equators of the $\mathrm{HgMn}$ stars with substantial rotation is sufficient to produce the observed pole-to-equator gradient in the $\mathrm{Hg}$ concentration. In addition to this axisymmetric effect, a range of hydrodynamical instabilities, possibly related to the rotationally induced mixing of the subphotospheric layers, may inhibit or amplify a buildup of the $\mathrm{Hg}$ overabundance in different surface zones, thereby creating a patchy time-dependent horizontal distribution of this chemical element. Detailed theoretical investigations into the $\mathrm{Hg}$ diffusion are needed to verify this hypothesis and establish timescales of the possible evolution of $\mathrm{Hg}$ distribution. A long-term observational campaign aimed at detecting such changes in the surface $\mathrm{Hg}$ maps also appears to be extremely promising both by itself and as a test of predictions of the radiative diffusion theory.

Acknowledgements. We acknowledge resources provided by the Vienna Atomic Line Database (VALD) and the SIMBAD database operated at the CDS, Strasbourg, France.

We also thank the referee, Dr. G. Wade, whose constructive remarks contributed to improvement of this paper.

\section{References}

Abt, H. A., Levato, H., \& Grosso, M. 2002, ApJ, 573, 359

Adelman, S. J., Gulliver, A. F., Kochukhov, O. P., \& Ryabchikova, T. A. 2002, ApJ, 575, 449

Barklem, P. S., Piskunov, N., \& O’Mara, B. J. 2000, A\&A, 363, 1091 
Chountonov, G. 2001, in Magnetic field across the HR diagram, ed. G. Mathys, S. Solanki, \& W. Wickramasinghe, ASP Conf. Ser., 248, 385

Donati, J.-F., Semel, M., Carter, B. D., Rees, D. E., \& Collier Cameron, A. 1997, MNRAS, 291, 658

ESA 1997, The Hipparchos Catalogue, ESA SP-1200

Glagolevskij, Yu. V., Romanyuk, I. I., Bychkov, V. D., \& Najdenov, I. D. 1985, Astron. Lett., 11, 45

Gray, D. F. 1992, The observation and analysis of stellar photospheres (Cambridge, New York: Cambridge University Press), 2nd ed.

Hauck, B., \& Mermilliod, M. 1998, A\&AS, 129, 431

Kupka, F., Piskunov N., Ryabchikova, T. A., Stempels, H. C., \& Weiss W. W. 1999, A\&AS, 138, 119

Kurucz, R. L. 1993, ATLAS9 Stellar Atmosphere Programs, CD-ROM No. 13 (Smithsonian Ap. Obs.)

Michaud, G., Charland, Y., \& Megessier, C. 1981, A\&A, 103, 244

Moon T. T., \& Dworetsky, M. M. 1985, MNRAS, 217, 305

Napiwotzki, R., Schoenberner, D., \& Wenske, V. 1993, A\&A, 268, 635

Panchuk, V. E., Klochkova, V. G., \& Najdenov, I. D. 1999, Preprint of the Spec. Astrophys. Obs., No. 135

Piskunov, N. 1992, in Stellar magnetism, ed. Yu. V. Glagolevskij, \& I. I. Romanyuk (St. Petersburg: Nauka), 92
Piskunov, N. E., \& Valenti, J. A. 2002, A\&A, 385, 1095

Pourbaix, D., Tokovinin, A. A., Batten, A. H., et al. 2004, A\&A, 424, 727

Proffitt, C. R., Brage, T., Leckrone, D. S., et al. 1999, ApJ, 512, 942

Rogers, N. Y. 1995, Comm. Asteroseismol., 78

Ryabchikova, T. A., Malanushenko, V. P., \& Adelman, S. J. 1999, A\&A, 351, 963

Schaller, G., Schaerer, D., Meynet, G., \& Maeder, A. 1992, A\&AS, 96, 269

Shorlin, S. L. S., Wade, G. A., Donati, J.-F., et al. 2002, A\&A, 392, 637

Socas-Navarro, H., Trujillo Bueno, J., \& Ruiz Cobo, B. 2000, ApJ, 530, 977

Stehlé, C. 1994, A\&AS, 104, 509

Strom, S. E., Wolff, S. C., \& Dror, D. H. A. 2003, AJ, 129, 809

Valenti, J. A., \& Piskunov, N. 1996, A\&AS, 118, 595

Vogt, S. S., \& Penrod, G. D. 1983, PASP, 95, 565

Wade, G. A., Abecassis, M., Auriere, M., et al. 2004, in Magnetic stars 2003, ed. Yu. V. Glagolevskij, D. O. Kudryavtsev, \& I. I. Romanyuk, 108

Woolf, V. M., \& Lambert, D. L. 1999, ApJ, 521, 414 